\title{
Ligand-Protein Interactions: A Hybrid ab initio/Molecular Mechanics Computational Study
}

\author{
Yornei R. Perez ${ }^{\mathrm{a}}$, Dinais Alvarez $^{\mathrm{a}}$, and Aldo F. Combariza ${ }^{*, \mathrm{a}}$
}

${ }^{\mathrm{a}}$ in silico Molecular Modeling and Computational Simulation Research Group, Department of Biology and Chemistry, Faculty of Education and Sciences, University of Sucre, Colombia

ªldo.combariza@unisucre.edu.co 


\begin{abstract}
The enzymes Cyclooxygenase (COX) or prostaglandin-endoperoxide synthase (PTGS) are important in the synthesis of prostaglandins, which are the main mediating chemi- cals at inflammatory processes. The body produces two highly homologous COX isoforms, cyclooxygenase-1 (COX-1) and cyclooxygenase-2 (COX-2). COX-1 is involved in the pro- duction of prostaglandins which take part in physiological processes such as: protection of the gastric epithelium, maintenance of renal flow, platelet aggregation, neutrophil mi- gration and also expressed in the vascular endothelium; Meanwhile COX-2 is inducible by proinflammatory stimuli. To counteract the symptoms of inflammation, nowadays is very frequent the use of nonsteroidal antiinflammatory drugs (NSAIDs); These drugs in addi- tion to other benefits, can also cause side effects on people's health (cardiovascular and respiratory problems, in the nervous system, among others). Due to the above, it is neces- sary to accelerate the investigations that allow to know in more detail the mechanisms of action that involve the use of natural plant products as pharmacological agents. In the pre- sent research, computational techniques are used, especially those based on the Molecular Docking Method to know the proteinligand interaction in systems of biological interest. It was possible to determine the structure-activity relationship in inflammatory processes involving the participation of a number of secondary plant metabolites such as luteolin, galangin, kaempferol, apigenin, morine and quercetin on the inactivation of the enzyme cyclooxygenase (COX-1 and COX -2). In the COX-1 / ligand coupling it was found that apigenin was the ligand which showed the lowest coupling energy with a value of -8.88 , whereas quercetin showed the highest value (-6.65); for the coupling COX-2 / ligand the apigenin also showed the lowest energy value (-8.93), while kaempferol showed the highest value (-7.51). This shows that apigenin is the ligand with the best stability within the active site of both enzymes. On the other hand, quercetin showed the highest relative selectivity with a protein-ligand selectivity index (PLSI) of 1.17, while kaempferol showed an PLSI of 0.91. Taking into account the parameters of stability and selectivity we can say that of all the ligands used, quercetin would be the ideal to block COX-2.
\end{abstract}

Keywords enzymes Cyclooxygenase, ligand, metabolites, antiinflammatory, Molecular Docking. 


\section{Introduction}

The awe-inspiring development of computational performance in the last decades has open the door to a new way of doing science, which is usually referred as computational experimentation or in silico experimentation. ${ }^{1-3}$ In general, an in silico experiment begins by modeling a real system using solid theoretical physical principles, followed by the simulation of the system through computer programmed mathematical algorithms. ${ }^{3-5}$

In this work, we use a hybrid ab initio/mechano-statistical technique to study the features of biological macromolecules, proteins, and their interactions with low molecular weight chemical species, called ligands. These protein-ligand biological systems, are a paradigmatic example of the structureactivity principle. ${ }^{5,6}$ We use here the Molecular Docking (MDock) technique, which allows us to predict the most appropriate conformation of a molecule (ligand) when it binds to another (protein) to form a stable complex. ${ }^{6}$ In fact, the use of MDock its seen as a advantageous technique in the study of protein-ligand interactions within the field of drug discovery and development. ${ }^{7-9}$

In this case, we intend to understand the structure-activity relationship of the ligand-protein interactions from an atomistic point of view and, therefore, to attain relevant information to disclose the effect of a biological active molecule, or ligand, on a specific proteic target. ${ }^{10}$ One relevant proteic target, very useful in the clinical research field, is the Prostaglandin-endoperoxide synthase (PTGS) or Cyclooxygenase (COX) enzyme. ${ }^{11,12}$ The first isoform is constitutive, and executes indispensable functions in the organism, while the second isoform intervenes in inflammatory processes, becoming the target of study for the search of novel anti-inflammatory compounds. ${ }^{13}$ Both, COX-1 and COX-2, have a high structural similarity, only differing by the relative position of three amino acids. This structural similarity brings about non-selectivity between COX-1 and COX-2, e.g., non steroidal antiinflammatory drugs (NSAIDs) are not selective for COX, which causes serious consequences in the organism. ${ }^{13,14}$

The lack of NSAIDs-COX selectivity, has fueled the search for new molecules or ligands. Sometimes these ligands are obtained from natural sources, that is, secondary metabolites from plants or animals, some of which are recognized to have a higher COX selectivity, helping to avoid or minimize the side effects produced by NSAIDs in the body. ${ }^{15,16}$

Within the secondary metabolites obtained from plants, we have a very wide group of chemicals 
known as flavonoids, which include: flavones, flavonols, antocyanidins and isoflavonols. ${ }^{17}$ These metabolites are recognized as anti-allergenic, anti-thrombotic, anti-oxidant and anti-inflammatory effects. ${ }^{18}$ Some examples of vegetal species which are used to extract these compunds are: Dioclea grandiflora, Sophora flavescens, Swinglea glutinosa and Muntingia calabura. ${ }^{15}$

The aim of this work is to use tools of computational biochemistry to describe the structureactivity relationship between COX-ligand systems, which is of paramount importance in inflammatory inhibition, involving the action of secondary metabolites.

\section{Methodology}

\section{COX structural characterization}

\section{COX-1}

From the Protein Data Bank (PDB) ${ }^{19}$, we have extracted 217 structures directly related with COX-1. These structures were discriminated according to the species from which they have been obtained, and are distributed as follows: Homo sapiens (51), Bos taurus (29), Mus musculus (25), Ovis aries (22), Escherichia coli (12), Rattus norvegicus (12), Zea mays (10), others species (53). ${ }^{19}$ Currently, there is a record of 171 ligands that form complexes with COX-1, among which are flurbiprofen, arachidonic acid, ibuprofen, sodium ion and nitric acid. ${ }^{19,20}$

\section{COX-2}

For COX-2, we have found 220 structures from the PDB, which were extracted from species like: Homo sapiens (51), Mus musculus (43), Bos taurus (28), Ovis aries (21), Escherichia coli (12), Rattus norvegicus (12), Saccharomyces cerevisiae (8), others (42). ${ }^{19}$ For this COX isoform, there are 178 ligands recognized, among which are the following: acetylsalicylic acid, flurbiprofen, urea, arachidonic acid and ibuprofen. ${ }^{20}$

For COX-1 we have used the model corresponding to Ovis aries (PDB ID: code 3N8Z) and for COX-2, the one forMus musculus (PDB ID: code 1CX2) and the experimental method used to dilucidate the tertiary structures of COX's was ray's x crystallography. ${ }^{21,23}$ These structures were selected because, according to Kurumbail et al, human COX-2 has a similarity of $87 \%$ with the murine enzyme, and the amino acid sequence that forms the active site is conserved between both species, 
therefore, we expect that human COX-2, and mainly the active site, will behave similarly to murine enzyme. ${ }^{23}$

\section{COX enzymes preparation and active sites identification.}

COX enzymes belongs to the oxidoreductase protein family, and forms complexes with selective inhibitors such as SC-558. ${ }^{23}$ pdb-files downloaded from the PDB-database, are provided as tetramer for COX-2 and as a dimer for COX-1. ${ }^{21}$ On the other hand, PyMOL ${ }^{26}$ was used to eliminate hemo groups, water molecules and ligands present in the structure of the enzyme.

\section{Ligands characterization}

The ChemSpider ${ }^{27}$ database was used to retrieve the chemical species of interest. ${ }^{28}$ These species are secondary metabolites coming from plants, belonging to the flavonoid family. We have selected the following metabolites as subjects of study:

- Apigenin (5,7-Dihydroxy-2-(4-hydroxyphenyl)-4H-chromen-4-one) ${ }^{29}$

- Luteolin (2-(3,4-Dihydroxyphenyl)-5,7-dihydroxy-4H-chromen-4-one) $)^{30}$

- Quercetin (2-(3,4-Dihydroxyphenyl)-3,5,7-trihydroxychromen-4-one) $)^{31}$

- Morin (2-(2,4-Dihydroxyphenyl)-3,5,7-trihydroxy-4H-chromen-4-one) ${ }^{32}$

- Galangin (3,5,7-Trihydroxy-2-phenyl-4H-chromen-4-one $)^{33}$

- Kaempferol (3,5,7-Trihydroxy-2-(4-hydroxyphenyl)-4H-chromen-4-one) ${ }^{34}$

\section{Geometry optimization}

Geometry optimization is a molecular modeling technique that allows us to obtain the structural conformations of lowest energy of a molecule by determining the critical points of the potential energy function and the rotations of chemical bonds that form the molecule. ${ }^{35,36}$ With this information we predict the tridimensional disposition of the atoms in a molecule creating a potential energy surface. ${ }^{35-39}$

MOPAC $^{40}$ was used to optimize the ligands chemical structures. MOPAC is a program based on the theoretical principles of quantum mechanics (QM), that is, the use of semi-empirical methods 
to predict properties of chemical structures and model chemical reactions. ${ }^{41} \mathrm{MNDO}, \mathrm{MINDO} / 3$, AM1 and PM3 were the Hamiltonians used to achieve molecular orbitals, heat of formation and its derivatives with respect to molecular geometry. ${ }^{41-44}$ Followed by the calculation of properties like: vibration spectra, thermodynamic quantities and constant forces that corresponds to molecules, radicals, ions and polymers. ${ }^{42,43}$ After the ligand optimization, we define parameters like the torsion center and the rotable bonds of the ligands.

\section{Molecular Docking}

Before the MDock process, we add the hydrogen bonds missing to COX structures. This step is carried out because the hydrogen atoms have a small nuclei and are very mobile, so they are not detected by the $\mathrm{X}$ rays in crystallographic process. AutoDock Vina (AUV) ${ }^{45}$ was used to behave the MDock Process. AUV uses a Lamarckian Genetic Algorithm (LGA) and a free-energy Empirical Punctuation Function (EPF) that allows a faster search method and provides reproducible results in larger systems. ${ }^{46-48}$ This software uses a semi-empiric force-field to evaluate the free-energy of conformations along the coupling simulations. At the same time, the force-field is parameterized using experimental information recover from numerous protein-inhibitor systems. ${ }^{46}$

We consider ten conformations for each protein-ligand interaction.

\section{Selectivity index and stability index}

We determine a index of selectivity and stability for each COX-ligand interaction. The stability index was determined by dividing each energetic value by the lowest energetic value. Whereas, the selectivity index was determined by dividing the binding energy of each ligand/COX-2 interaction between the binding energy of each ligand-COX-1 interaction, taking into account the correspondence between the ligands. Finally, the values obtained were normalized.

\section{Results and Discussion}

\section{Molecular characterization of COX}

The tertiary structures of COX are very similar, thus, they share the same folding units. Because of this, we use the COX-1 structure to characterize both enzymes. We can divide the tertiary structure 
of COX-1 in three folding units, showed in Figure 1.

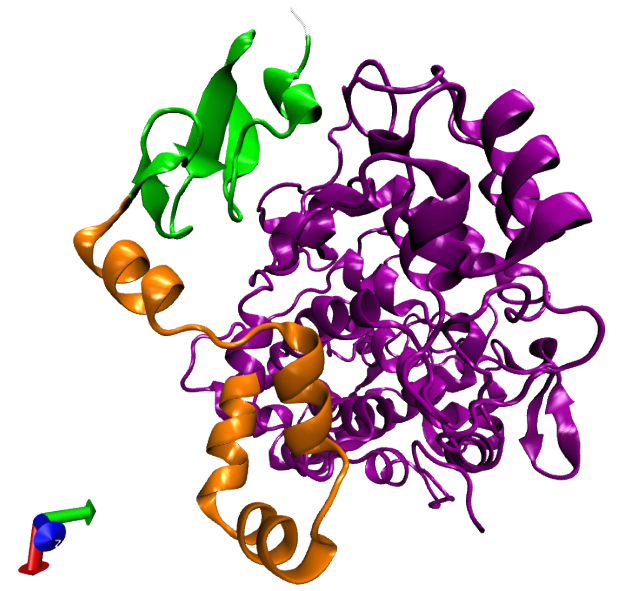

Figure 1: Folding units of COX-1. First unit is show in green, the second is show in orange and the third is show in violet.

The first folding unit is formed by residues 34-72 in positions amino-terminal and form a small compact domain binded by three disulfide bonds, as Figure 2A shows. The global conformation of this domain, including the disposition of the disulfide bonds, is very similar to the conformation of the epidermal growth factor and binds covalently to the principal chain of the enzyme by another disulfide bridge, that is, Cys-37-Cys-159. The second unit is formed by residues 73-116 and form a structure of alpha helix A, B, C and D that are located at one side of the first folding unit, as we illustrate in Figure 2B. These alpha helix are highly amphipathic and represents a structural motif for the insertion of enzyme to the lipid bilayer. And the last folding unit consist of a large catalytic globular domain formed by residues 117-587. This catalytic domain is a globular structure that contains the cyclooxygenase and peroxidase active sites. Both sites, peroxidase and cyclooxygenase, comprise two differents lobes in the polypeptidic chain although interlaced with each other. The largest lobe is made by a structure formed by seven alpha helices, whereas the smallest lobe of the catalytic domain is formed by six alpha helices. These alpha helices form a set, more or less parallel, with its axes (see Figure 2C). 
(a)

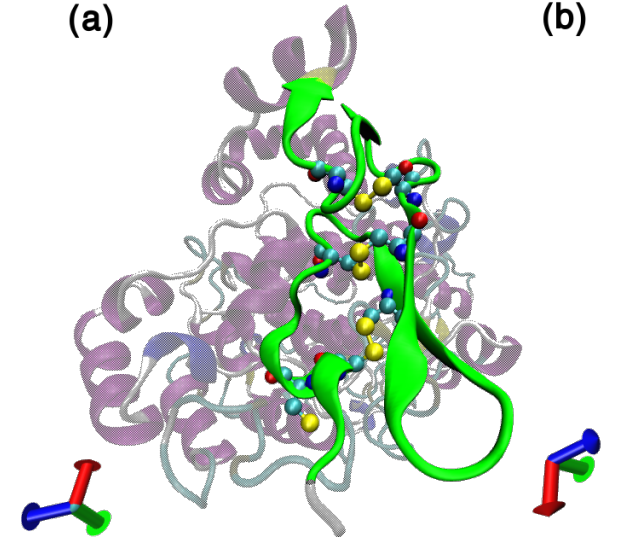

(b)

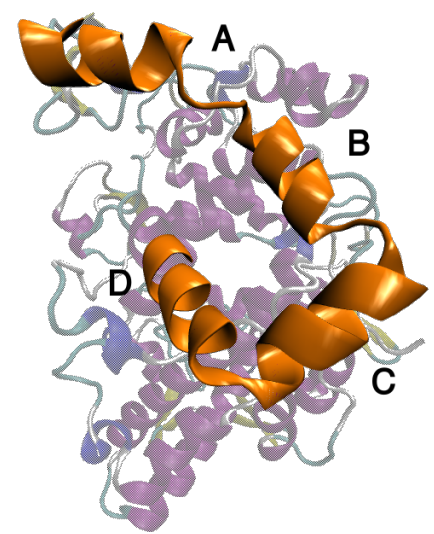

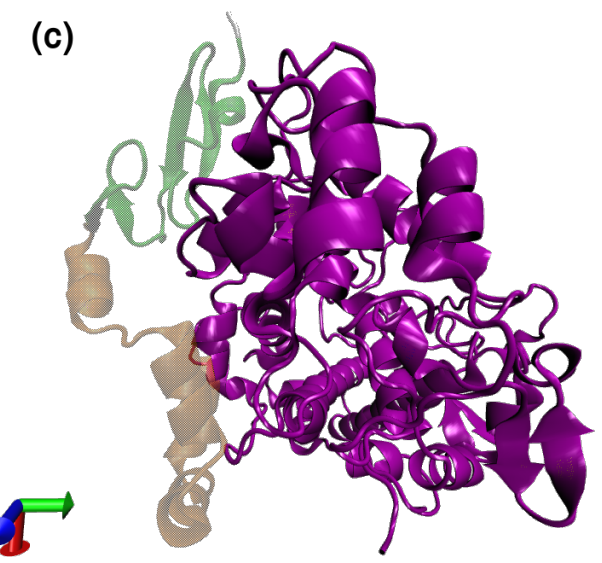

Figure 2: Folding units of COX-1. (a) first folding unit in green and the three disulfide bonds are shown in yellow; (b) in orange is show the second folding unit and the four alpha helices (A, B, C and D); (c) in violet, the third folding unit is represent by alpha helices and beta sheets.

Characterization of the secondary structures of COX

Table 1: COX-1 secondary structure characterization

\begin{tabular}{ccccc}
\hline & Coil & 3-10 Helix & Alpha helix & Beta sheet \\
\hline $32-34$ & 324 & $35-38$ & $74-81$ & $46-50$ \\
$39-41$ & $354-362$ & $281-283$ & $86-93$ & $54-58$ \\
63 & $370-377$ & $363-366$ & $97-105$ & $64-65$ \\
70 & $391-394$ & $388-390$ & $107-121$ & $71-72$ \\
73 & 403 & $498-500$ & $139-143$ & $130-131$ \\
$82-85$ & 411 & $538-540$ & $174-182$ & $149-150$ \\
$94-96$ & 418 & $547-550$ & $196-207$ & $245-248$ \\
106 & $428-429$ & & $238-244$ & $251-252$ \\
129 & 431 & & $296-319$ & $255-257$ \\
$135-138$ & $433-439$ & & $325-353$ & $260-262$ \\
148 & $459-462$ & & $379-385$ & $395-397$ \\
$154-155$ & $471-477$ & & $404-407$ & $400-402$ \\
167 & $483-484$ & & $412-417$ & \\
$172-173$ & $496-497$ & & $419-427$ & \\
188 & $501-502$ & & $445-458$ & \\
211 & $510-511$ & & $463-470$ & \\
$236-237$ & $517-518$ & & $478-482$ & \\
$253-254$ & 537 & $485-495$ & \\
$263-264$ & 546 & & $503-509$ & \\
$271-275$ & $551-552$ & & $520-536$ & \\
280 & $562-563$ & & $553-561$ & \\
284 & 571 & $564-570$ & \\
286 & $576-580$ & & & \\
& $582-584$ & & &
\end{tabular}


Table 2: COX-2 secondary structure characterization

\begin{tabular}{ccccc}
\hline & Coil & 3-10 Hélices & Hélices alfa & Láminas Beta \\
\hline 33 & $263-264$ & $363-366$ & $74-81$ & $46-49$ \\
40 & $271-275$ & $388-390$ & $86-94$ & $55-58$ \\
63 & 324 & $498-500$ & $97-104$ & $64-65$ \\
70 & $354-362$ & $538-540$ & $106-121$ & $71-72$ \\
73 & $370-377$ & & $139-143$ & $245-248$ \\
$82-85$ & 391 & & $174-182$ & $251-252$ \\
$95-96$ & 396 & $196-209$ & $255-257$ \\
105 & 401 & $238-243$ & $260-262$ \\
$129-130$ & 403 & $296-317$ & \\
138 & 418 & & $325-353$ & \\
148 & $428-431$ & & $379-384$ & \\
$154-155$ & $437-439$ & & $404-407$ & \\
167 & $459-461$ & $411-417$ & \\
$172-173$ & $471-477$ & $419-427$ & \\
188 & $483-484$ & & $444-458$ & \\
195 & 501 & & $463-470$ & \\
$210-212$ & $510-511$ & $478-482$ & \\
$236-237$ & $561-563$ & $485-495$ & \\
244 & $576-580$ & $503-509$ & \\
$253-254$ & & $520-533$ & \\
& & $553-560$ & \\
\hline
\end{tabular}

Topological and molecular characterization of the active sites of COX

The active sites of COX enzymes have a high similarity. Both active sites consist of a hydrophobic channel with an approximate extension of 25 Angstroms. This channel originates in the Membrane Binding Domain (MBD) and extends to the nucleus of the globular domain. ${ }^{21-23}$ The amino acids covering the hydrophobic channel of COX active sites are: Leu117, Arg120, Val434 (COX2), Ile434 (COX-1), Phe205, Phe209, Val344, Ile345, Tyr348, Val349, Leu352, Ser353, Tyr355, Leu359, Phe381, Leu384, Tyr385, Trp387, Phe518, Ile523 (COX-1), Val523 (COX-2), Gly526, Ala527, Ser530, His513 (COX-1), Arg513 (COX-2), Leu531, Gly533, Leu534 and these are shown in Figure 3. Only three of the channel residues are polar Arg120, Ser353 y Ser530.
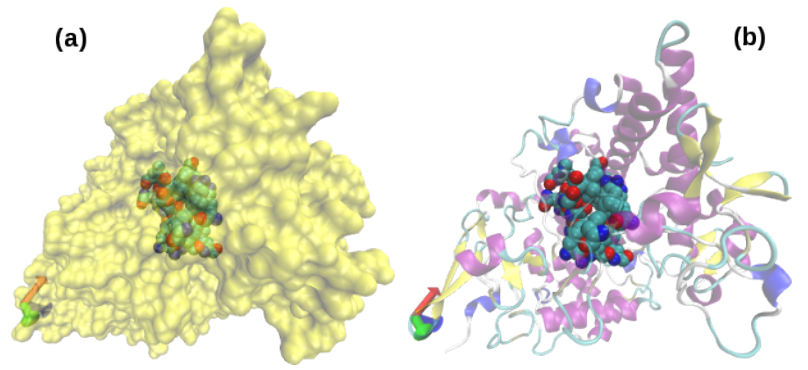

Figure 3: Amino acids of COX active site. (a) In the center of the figure are shown the amino acids that form the active site of the enzyme, highlighting the third folding unit. 
The residue Ser530 is acetylated by aspirin, while the residue Arg120 binds to the carboxylate groups of the fatty acids and many NSAIDs. ${ }^{49-52}$

The size of the active site of COX-2 is approximately about $20 \%$ more large that the COX-1 enzyme. This difference in the active sites of COX is very important due to the variation of three residues at positions 434, 513 and 523 of both enzymes, as we show in Figure 4. The variation in the size of both active sites has been an important feature for the development of specific NSAIDs for COX-2. ${ }^{53}$ Figure 5 shows, an superposition of the active sites of both enzymes with the residues that differentiate them, at the same point.
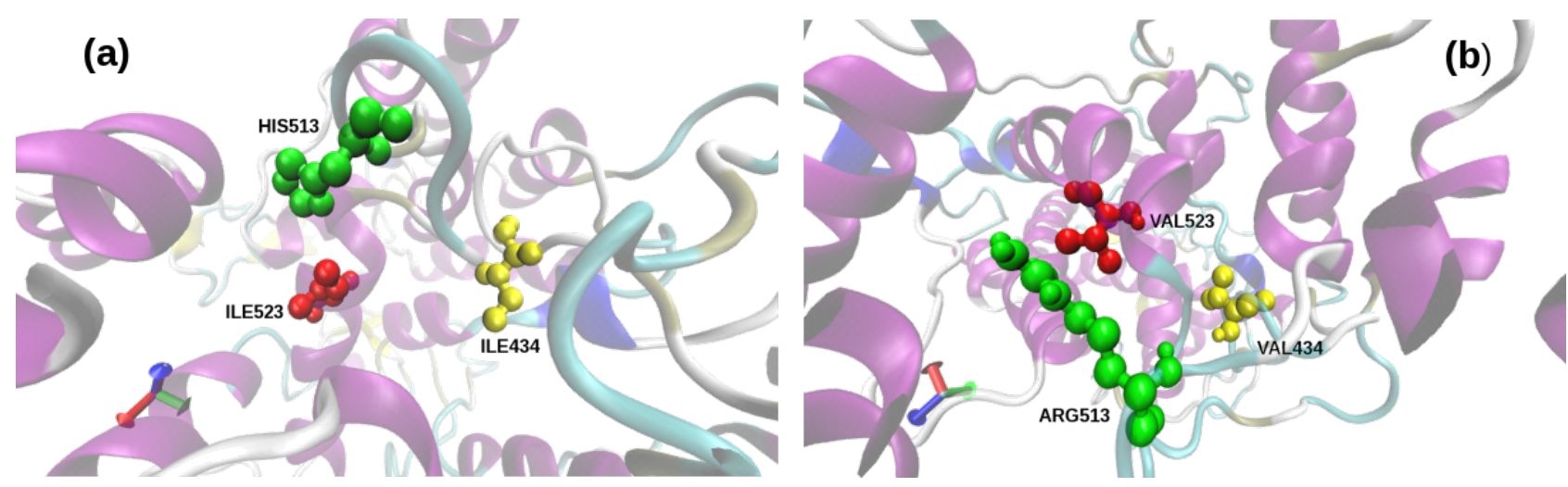

Figure 4: Amino acids that differentiate the active sites of COX. A. Amino acid Ile434 is highlighting in yellow, His513 in green and Ile523 in red to COX-1. B. Amino acids of COX-2 are highlighting as: Val434 in yellow, Arg513 in green and Val523.
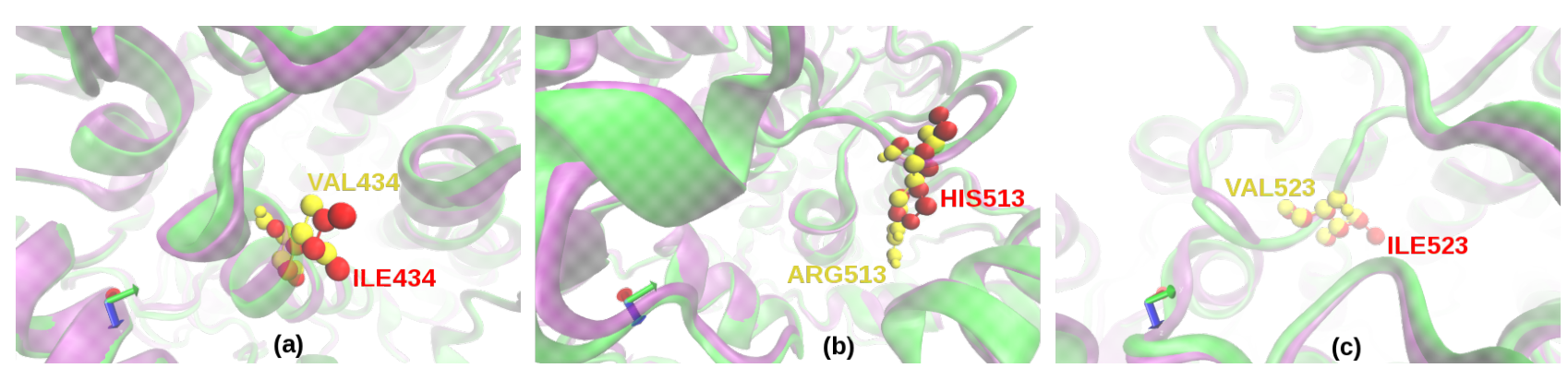

Figure 5: Superposition of COX active sites. COX-1: green, COX-2: violet. (a) isoleucin for COX-1 and valine for COX-2; (b) histidin for COX-1 and arginin for COX-2; (c) isoleucin for COX-1 and valin for COX-2.

\section{Geometry optimization}

Table 3 shows the values obtained from the calculation of minimization energies and the calculation of molecular orbitals HOMO and LUMO of each ligand. These values are ordered according to the HOMO energy from the best nucleophile to the best electrophile. The HOMO orbital is the last orbital that is doubly occupied and shows where the pair of electrons most susceptible to electrophilic 
attacks is located. Taking into account the values shown in Table 1, we can see that, of all the ligands, galangin is the best nucleophile, while apigenin is the worst nucleophile.

Table 3: Minimization energy values and molecular orbitals HOMO and LUMO

\begin{tabular}{ccccccc}
\hline Ligand & Galangin & Kaempferol & Quercetin & Morin & Luteolin & Apigenin \\
\hline Total Energy & -3452.9 & -3748.7 & -4044.0 & -4043.9 & -3749.0 & -3453.7 \\
HOMO & -8.974 & -9.075 & -9.099 & -9.147 & -9.351 & -9.458 \\
LUMO & -0.755 & -0.997 & -1.084 & -0.8 & -1.18 & -1.071 \\
\hline
\end{tabular}

It should be noted that the calculations of the energies of the molecular orbitals HOMO and LUMO was made to know the distribution of the electrical charges along the molecule. Therefore, interactions with electrophilic groups are controlled by the HOMO orbital, while the nucleophilic attacks are controlled by the LUMO orbitals. Thus, Figure 6 allow us to observe, in a spatial way, the distribution of the charges and the regions of the molecule with high electron density, that is, regions susceptible to electrophilic and nucleophilic attacks. These areas also show the electrostatic potential of the molecules, the red color shows the negative part and the blue color shows the positive part. The green and yellow regions show the poorest electron zones.
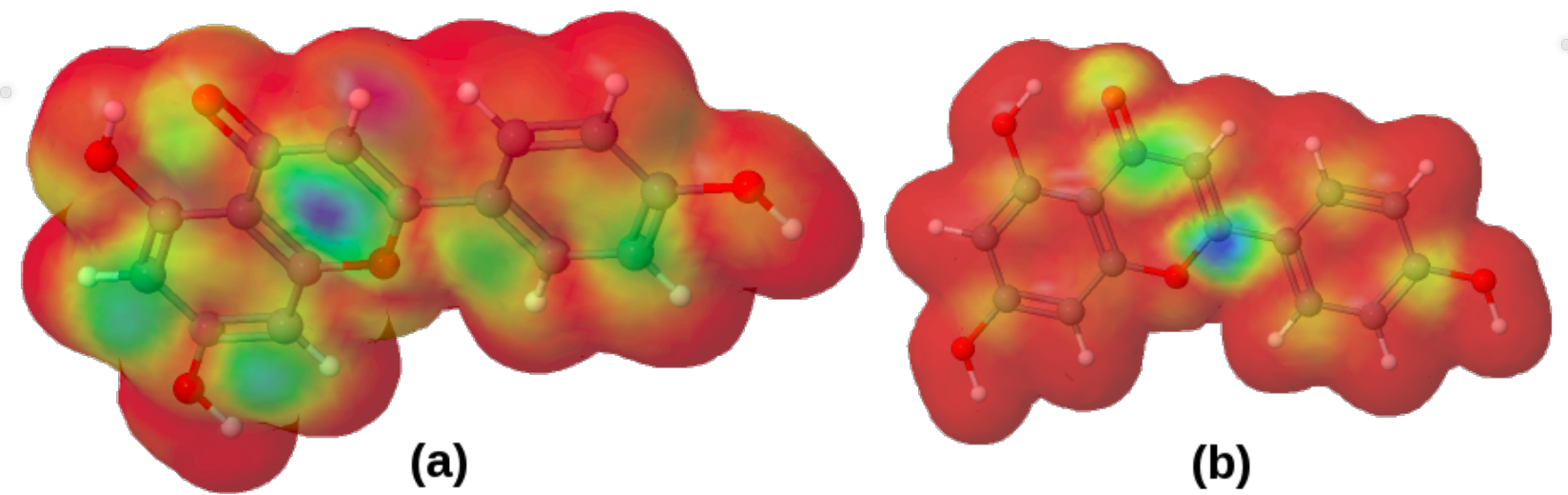

Figure 6: Apigenin electronic density maps. (a) HOMO orbital: in red, the zone most susceptible to electrophilic attacks; (b) LUMO orbital: the region most susceptible to nucleophilics attacks.

On the other hand, Table 4 shows the optimization energy values of each ligand in gaseous state, that is, before the coupling process, as well as the optimization energies after the coupling process and the energy differences for the COX-1/ligand interaction. Table 5 shows the values corresponding to COX-2/ligand interactions. 
Table 4: Optimization and pos-coupling energies of COX-1/ligand complex.

\begin{tabular}{ccccccc}
\hline \multicolumn{7}{c}{ COX-1 } \\
\hline Ligand & Luteolin & Galangin & Apigenin & Kampferol & Morin & Quercetin \\
\hline Optimization Energy & -3749.02 & -3452.95 & -3453.72 & -3748.74 & -4043.90 & -4044.03 \\
MOPAC Energy & -3746.46 & -3452.89 & -3307.05 & -3745.36 & -4042.01 & -4043.43 \\
Energy Difference & -2.56 & -0.05 & -146.67 & -3.38 & -1.89 & -0.60 \\
\hline
\end{tabular}

Table 5: Optimization and pos-coupling energy values of COX-2/ligand complex

\begin{tabular}{ccccccc}
\hline \multicolumn{7}{c}{ COX-2 } \\
\hline Ligand & Luteolin & Galangin & Apigenin & Kaempferol & Morin & Quercetin \\
\hline Optimization Energy & -3749.02 & -3452.95 & -3453.72 & -3748.74 & -4043.90 & -4044.03 \\
MOPAC Energy & -3746.82 & -3452.80 & -3449.55 & -3745.36 & -4041.95 & -4042.41 \\
Energy Difference & -2.20 & -0.15 & -4.18 & -3.38 & -1.95 & -1.63 \\
\hline
\end{tabular}

\section{Molecular Docking}

For each protein-ligand complex, we selected ten different conformations and for each one we evaluated the binding energy of the complex formed. Table 6 shows the interaction energy for each conformation of Galangin/COX-1 system, the amino acids and hydrogen bonds involved. In all conformations there is formation of at least one hydrogen bonds, except the conformation 9 where there is formation of 3 hydrogen bonds. The conformation number nine have the lowest energetic values, which means that in this conformation the ligand have more stability in the active site of enzyme that others conformations. 
Table 6: COX-1/Galangin complex. 1-10: ten conformations obtained with each one of the energetic values X: amino acids that interact with the ligand in each conformation. From the line upwards: presence of hydrogen bonds. Highlighted in green: amino acids that interact with the ligand and that are repeated in the indicated conformations.

\begin{tabular}{|c|c|c|c|c|c|c|c|c|c|c|}
\hline \multirow[t]{2}{*}{ Amino acids } & \multicolumn{10}{|c|}{ Conformations } \\
\hline & 1 & 2 & 3 & 4 & 5 & 6 & 7 & 8 & 9 & 10 \\
\hline TYR 355 & $\mathrm{X}$ & $\mathrm{X}$ & $\mathrm{X}$ & $\mathrm{X}$ & $\mathrm{X}$ & $\mathrm{X}$ & $\mathrm{X}$ & $\mathrm{X}$ & $\mathrm{X}$ & $\mathrm{X}$ \\
\hline SER 530 & $X$ & $X$ & $\mathrm{X}$ & $\mathrm{X}$ & $X$ & $X$ & $X$ & $\mathrm{X}$ & $X$ & $X$ \\
\hline ILE 523 & $\mathrm{X}$ & $\mathrm{X}$ & $X$ & $\mathrm{X}$ & $X$ & $X$ & $X$ & $X$ & $\mathrm{X}$ & $X$ \\
\hline MET 522 & $X$ & $X$ & $X$ & $X$ & $\mathrm{X}$ & $\mathrm{X}$ & $\mathrm{X}$ & $\mathrm{X}$ & $\mathrm{X}$ & $X$ \\
\hline LEU 531 & $\mathrm{X}$ & $\mathrm{X}$ & $\mathrm{X}$ & $\mathrm{X}$ & $X$ & $X$ & $X$ & $X$ & $X$ & $X$ \\
\hline VAL 349 & $\mathrm{X}$ & $X$ & $\mathrm{X}$ & $\mathrm{X}$ & $\mathrm{X}$ & $\mathrm{X}$ & $\mathrm{X}$ & $\mathrm{X}$ & $\mathrm{X}$ & $\mathrm{X}$ \\
\hline ALA 527 & $X$ & $\mathrm{X}$ & $X$ & $\mathrm{X}$ & $\mathrm{X}$ & $X$ & $\mathrm{X}$ & $X$ & $\mathrm{X}$ & $\mathrm{X}$ \\
\hline TRP 387 & $X$ & & $X$ & $\mathrm{X}$ & $\mathrm{X}$ & $X$ & $\mathrm{X}$ & $\mathrm{X}$ & $\mathrm{X}$ & $\mathrm{X}$ \\
\hline SER 353 & $\mathrm{X}$ & $\mathrm{X}$ & $X$ & $\mathrm{X}$ & $\mathrm{X}$ & $\mathrm{X}$ & $\mathrm{X}$ & $\mathrm{X}$ & & $\mathrm{X}$ \\
\hline LEU 352 & $\mathrm{X}$ & $\mathrm{X}$ & $\mathrm{X}$ & $\mathrm{X}$ & $\mathrm{X}$ & $X$ & $X$ & $\mathrm{X}$ & & $X$ \\
\hline TYR 385 & $\mathrm{X}$ & & $\mathrm{X}$ & $\mathrm{X}$ & $\mathrm{X}$ & $X$ & $X$ & $\mathrm{X}$ & & $X$ \\
\hline PHE 518 & & $X$ & & $X$ & $\mathrm{X}$ & $X$ & $X$ & $X$ & $\mathrm{X}$ & $X$ \\
\hline GLY 526 & & $\mathrm{X}$ & & & & & & & $\mathrm{X}$ & \\
\hline TYR 348 & $\mathrm{X}$ & & $\mathrm{X}$ & & & & & & & \\
\hline PHE 381 & & $X$ & & & & & & & & \\
\hline ARG 120 & & & & & & & & & $\mathrm{X}$ & \\
\hline VAL 116 & & $\mathrm{X}$ & & & & & & & & \\
\hline LEU 359 & & $X$ & & & & & & & & \\
\hline LEU 384 & & & & & & & & & $\mathrm{X}$ & \\
\hline Bind energy & -6.96 & -6.96 & -6.97 & -7.2 & -7.18 & -7.19 & -7.18 & -7.18 & -7.26 & -7.18 \\
\hline
\end{tabular}

\section{Most stable configuration}

The selection criteria of the most stable configuration for each protein-ligand coupling, was based on energetic values, that is, the lowest interaction energy indicate the highest stability of the complex.

For each of the 6 protein-ligands pairs, the best conformation based on the aforementioned energy terms was selected. The binding energy values between all the ligands were compared in order to determine which of them best matches the active site of the protein. Table 7 shows the best conformation 
for each COX-1/ligand pair and the amino acids that interact with the ligand in each conformation. The interaction is remarkable by hydrogen bonds, attraction forces, electrostatic interactions and Van der Waals dispersion forces. Table 8 shows this information for COX-2/ligand system.

Table 7: Best conformation of COX-1/ligand complex

\begin{tabular}{|c|c|c|c|c|c|c|}
\hline \multicolumn{7}{|c|}{ COX-1 } \\
\hline Ligand & Apigenin & Kaempferol & Luteolin & Morin & Galangin & Quercetin \\
\hline Conformations & $1-10$ & 1 & 5 & 6 & 9 & 3 \\
\hline \multirow[t]{7}{*}{ Binding energy } & -8.88 & -8.24 & -7.79 & -7.71 & -7.26 & -6.65 \\
\hline & TRP 387 & TRP 387 & TRP 387 & TRP 387 & TRP 387 & TRP 387 \\
\hline & VAL 349 & HIS 388 & VAL 349 & LEU 390 & VAL 349 & VAL 349 \\
\hline & SER 530 & HIS 386 & SER 530 & PHE 210 & SER 530 & SER 530 \\
\hline & ILE 523 & LEU 390 & ILE 523 & ALA 202 & ILE 523 & ILE 523 \\
\hline & GLY 526 & MET 391 & LEU 384 & GLN 203 & GLY 526 & TYR 355 \\
\hline & ALA 527 & TYR 385 & TYR 385 & HIS 207 & MET 522 & SER 353 \\
\hline \multirow[t]{7}{*}{ Amino acids } & LEU 384 & ASN 382 & MET 522 & ASN 382 & ALA 527 & TYR 385 \\
\hline & LEU 531 & ALA 202 & ALA 527 & TYR 385 & LEU 531 & LEU 352 \\
\hline & MET 522 & THR 206 & ARG 120 & THR 206 & PHE 518 & VAL 116 \\
\hline & LEU 352 & HIS 207 & VAL 116 & HIS 388 & ARG 120 & TYR 348 \\
\hline & ARG 120 & PHE 210 & PHE 381 & HIS 386 & LEU 384 & ARG 120 \\
\hline & TYR 355 & & PHE 518 & & TYR 355 & MET 522 \\
\hline & VAL 116 & & LEU352 & & & ALA527 \\
\hline
\end{tabular}

Apigenin shows the lowest coupling energy in complex with COX-1, which means that this ligand have an advantage in energetic terms that others. Apigenin interact with the same amino acids in all conformations of the COX-1/Apigenin system and have the same energetic values. The ligands that follows Apigenin in terms of high energetic stability are: Kaempferol, Luteolin, Morin, Galangin and Quercetin, repectively. 
Table 8: Best conformation of COX-2/ligand complex

\begin{tabular}{|c|c|c|c|c|c|c|}
\hline \multicolumn{7}{|c|}{$\mathrm{COX}-2$} \\
\hline Ligand & Apigenine & Kaempferol & Luteolin & Morin & Galangin & Quercetin \\
\hline Conformations & 7 & 3 & 1 & 1 & 3 and 8 & 1 and 9 \\
\hline Binding energy & -8.93 & -7.51 & -7.58 & -7.91 & -7.55 & -7.76 \\
\hline \multirow{13}{*}{ Amino acids } & SER 530 & SER 353 & VAL 523 & HIS 90 & TYR 355 & TYR 355 \\
\hline & ALA 378 & VAL 523 & TYR 355 & SER 353 & VAL 523 & VAL 5239 \\
\hline & ILE 377 & HIS 90 & SER 530 & VAL 349 & HIS 90 & SER 530 \\
\hline & PHE 381 & LEU 352 & LEU 352 & LEU 352 & SER 353 & TYR 385 \\
\hline & PHE 209 & TYR 355 & TYR 385 & SER 530 & LEU 352 & LEU 352 \\
\hline & PHE 205 & PHE 518 & ALA 527 & VAL 523 & ALA 527 & SER 353 \\
\hline & VAL 228 & ILE 517 & HIS 90 & TYR 355 & PHE 518 & GLN 192 \\
\hline & LEU 534 & GLN 192 & ARG 513 & MET 522 & ALA 516 & HIS 90 \\
\hline & GLY 533 & ALA 516 & TYR 348 & LEU 384 & ILE 517 & PHE 518 \\
\hline & ASN 375 & GLY 526 & SER 353 & TYR 385 & GLN 192 & ALA 527 \\
\hline & PHE 529 & PHE 381 & & TRP387 & & \\
\hline & LYS 532 & & & PHE 518 & & \\
\hline & ILE 124 & & & & & \\
\hline
\end{tabular}

Table 8 shows that conformation 7 (Apignenin) have the lowest value of coupling energy, namely, is the most stable conformation of the ligand in the active site of enzyme in the coupling process. Galangin and Quercetin shows 2 conformations with the same amino acids and the same values of coupling energy. In this case, the ligands that follows Apigenin in high stability are: Morin, Quercetin, Luteolin, Galangin and Kaempferol, respectively. On the other hand, electrostatic terms has been the more relevants energetic terms to choose the conformations and the more stable ligands.

Figure 7 shows, highlighted in red, five zones of high electronic density of Apigenin, in which the hydroxyl groups of the molecule are found. Due to the high electronic density, the formation of electrostatic interactions is possible. The amino acids that interact with Apigenin are: SER 530, ALA 378, ILE 377, PHE 381, PHE 209, PHE 205, VAL 228, LEU 534, GLY 533, ASN 375, PHE 529, 
LYS 532 and ILE 124. There is formation of a hydrogen bond between the amino group of ALA 378 and a hydroxyl group (OH-) of Apigenin, which helps to stabilize said conformation.

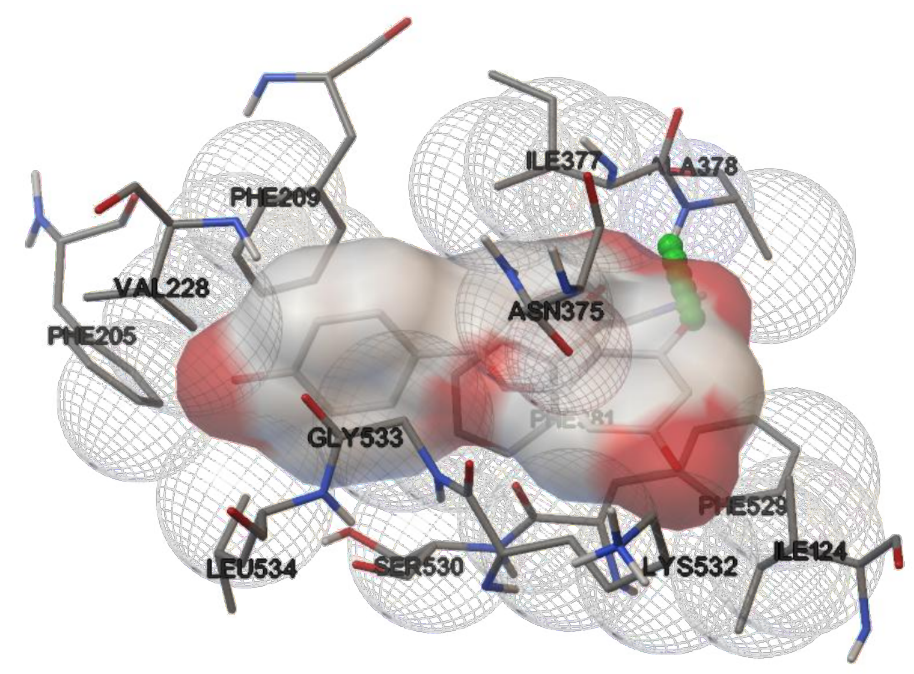

Figure 7: COX-2/Apigenin complex. The ligand is surrounded by amino acids that participate in the interaction with the active site of the enzyme. A hydrogen bond is shown in green.

Table 8 shows a comparison between the COX-1/ligand and COX-2/ligand interactions. Following the energetic terms, Apigenin have the most high stability in the active site of COX-1 with a value of -8.88 and a value of -8.93 in COX-2. While, the comparison of the coupling energy of Apigenin in COX-1 and COX-2, it shows that the stability is higher in the COX-1/Apigenin system.

Table 9: Comparison of ligand/COX-1 system and ligand/COX-2 system

\begin{tabular}{|c|c|c|c|c|c|c|c|c|c|c|c|c|}
\hline \multirow{2}{*}{$\begin{array}{c}\text { Ligand } \\
\text { Enzymes }\end{array}$} & \multicolumn{2}{|c|}{ Apigenin } & \multicolumn{2}{|c|}{ Galangin } & \multicolumn{2}{|c|}{ Kaempferol } & \multicolumn{2}{|c|}{ Luteolin } & \multicolumn{2}{|c|}{ Morin } & \multicolumn{2}{|c|}{ Quercetin } \\
\hline & COX-1 & COX-2 & COX-1 & COX-2 & COX-1 & COX-2 & COX-1 & COX-2 & $\mathrm{COX}-1$ & $\mathrm{COX}-2$ & $\mathrm{COX}-1$ & $\mathrm{COX}-2$ \\
\hline Bind energy & -8.88 & -8.93 & -7.26 & -7.55 & -8.24 & -7.51 & -7.79 & -7.58 & -7.71 & -7.91 & -6.65 & -7.76 \\
\hline \multirow{13}{*}{ Amino acids } & TRP 387 & SER 530 & TRP 387 & TYR 355 & TRP 387 & SER 353 & TRP 387 & VAL 523 & TRP 387 & HIS 90 & TRP 387 & TYR 355 \\
\hline & VAL 349 & ALA 378 & VAL 349 & VAL 523 & HIS 388 & VAL 523 & VAL 349 & TYR 355 & LEU 390 & SER 353 & VAL 349 & VAL 523 \\
\hline & SER 530 & ILE 377 & SER 530 & HIS 90 & HIS 386 & HIS 90 & SER 530 & SER 530 & PHE 210 & VAL 349 & SER 530 & SER 530 \\
\hline & ILE 523 & PHE 381 & ILE 523 & SER 353 & LEU 390 & LEU 352 & ILE 523 & LEU 352 & ALA 202 & LEU 352 & ILE 523 & TYR 385 \\
\hline & GLY 526 & PHE 209 & GLY 526 & LEU 352 & MET 391 & TYR 355 & LEU 384 & TYR 385 & GLN 203 & SER 530 & TYR 355 & LEU 352 \\
\hline & ALA 527 & PHE 205 & MET 522 & ALA 527 & TYR 385 & PHE 518 & TYR 385 & ALA 527 & HIS 207 & VAL 523 & SER 353 & SER 353 \\
\hline & LEU 384 & VAL 228 & ALA 527 & PHE 518 & ASN 382 & ILE 517 & MET 522 & HIS 90 & ASN 382 & TYR 355 & TYR 385 & GLN 192 \\
\hline & LEU 531 & LEU 534 & LEU 531 & ALA 516 & ALA 202 & GLN 192 & ALA 527 & ARG 513 & TYR 385 & MET 522 & LEU 352 & HIS 90 \\
\hline & MET 522 & GLY 533 & PHE 518 & ILE 517 & THR 206 & ALA 516 & ARG 120 & TYR 348 & THR 206 & LEU 384 & VAL 116 & PHE 518 \\
\hline & LEU 352 & ASN 375 & ARG 120 & GLN 192 & HIS 207 & GLY 526 & VAL 116 & SER 353 & HIS 388 & TYR 385 & TYR 348 & ALA 527 \\
\hline & ARG 120 & PHE 529 & LEU 384 & & PHE 210 & PHE 381 & PHE 381 & & HIS 386 & TRP 387 & ARG 120 & \\
\hline & TYR 355 & LYS 532 & TYR 355 & & & & PHE 518 & & & PHE 518 & MET 522 & \\
\hline & VAL 116 & ILE 124 & & & & & LEU 352 & & & & ALA 527 & \\
\hline
\end{tabular}




\section{Stability Index SI}

We have been established a stability index according to the energy values (bind energies) of each protein-ligand interaction. We quantified the force exerted by the interaction of the ligand with the active site of enzyme. The interaction index is defined as follows:

$$
I E=\frac{E_{i}}{E_{\text {minor }}}
$$

Where, $E_{i}$ represent the bind energy of each protein-ligand interaction and $E_{\text {minor }}$ is the minor bind energy of all protein-ligand interaction. The IE is normalized as follows:

$$
I E N=\frac{\left(E_{j}-E_{\text {minor }}\right)}{\left(E_{\text {mayor }}-E_{\text {minor }}\right.}
$$

Where, IEN is the normalized stability index, $E_{i}$ represent the bind energy of each protein-ligand interaction, $E_{\text {minor }}$ is the minor bind energy of all protein-ligand interaction and $E_{\text {higher }}$ is the higher bind energy of all protein-ligand interactions.

Figure 8(a) represent the greater stability of Apigenin in the active site of COX-1, followed by Kaempferol, Luteolin, Morin, Galangin and Quercetin with the lowest stability. While, Figure 8(b) shows that Apigenin was the ligand more stable in the active site of COX-2, followed by Morin, Quercetin, Luteolin, Galangin and Kaempferol. In the region of the ligands with the lowest stability, that is, 0.51, we find Luteolin, Morin, Galangin and Quercetin.
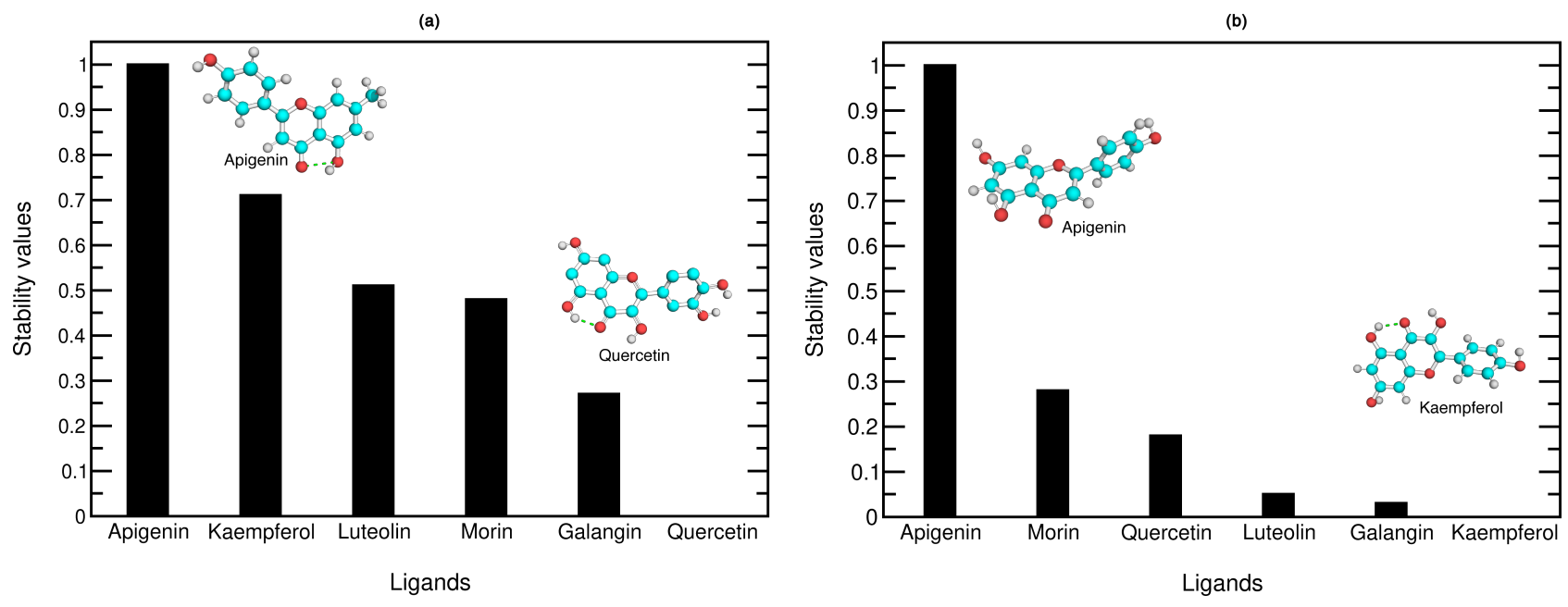

Figure 8: Selectivity index of ligands. (a) selectivity index for COX-1; (b) selectivity index for COX-2.

Quercetin have the lowest relative stability index of all ligands in complex with COX-1, while 
the same ligand has a higher stability index in complex with COX-2 (IE 0.18), which means that this ligand can be the structure with more energetic differences between both active sites.

On the other hand, Kaempferol have the higher stability index in COX-1 (IE 0.71) while this index is close to zero in COX-2. This suggest that Kaempferol is in a low energy state in the active site of COX-1. Table 10 shows a comparison of the energetic parameters and the formation of interactions between the ligands with IE values more representatives.

Table 10: Energetic parameters and hydrogen bonds formation of Apigenin, Quercetin and Kaempferol.

\begin{tabular}{ccccccc}
\hline Ligand & \multicolumn{2}{c}{ Apigenin } & \multicolumn{2}{c}{ Quercetin } & \multicolumn{2}{c}{ Kaempferol } \\
\hline Enzymes & COX-1 & COX-2 & COX-1 & COX-2 & COX-1 & COX-2 \\
\hline Heat of Formation kJ mol & -362.63 & -235.67 & -917.04 & -813.97 & -467.72 & -469.34 \\
Total Energy eV & -3307.05 & -3449.55 & -4043.43 & -4042.41 & -3745.36 & -3745.36 \\
Electronic Energy eV & -21346.54 & -21448.13 & -25951.83 & -25836.36 & -23672.59 & -23641.45 \\
Core-Core Repulsion eV & 18039.48 & 17998.58 & 21908.39 & 21793.95 & 19927.22 & 19896.09 \\
Ionization Potential eV & 9.21 & 9.52 & 9.17 & 9.09 & 9.06 & 9.17 \\
HOMO Energy eV & -9.21 & -9.52 & -9.17 & -9.09 & -9.06 & -9.17 \\
LUMO Energy eV & -1.36 & -0.99 & -1.21 & -1.37 & -1.53 & -1.44 \\
\hline
\end{tabular}

Figure 9 shows the interaction of Apigenin, Quercetin and Kamepferol with COX, also highlighted the amino acids of active sites and the hydrogen bonds formed. 

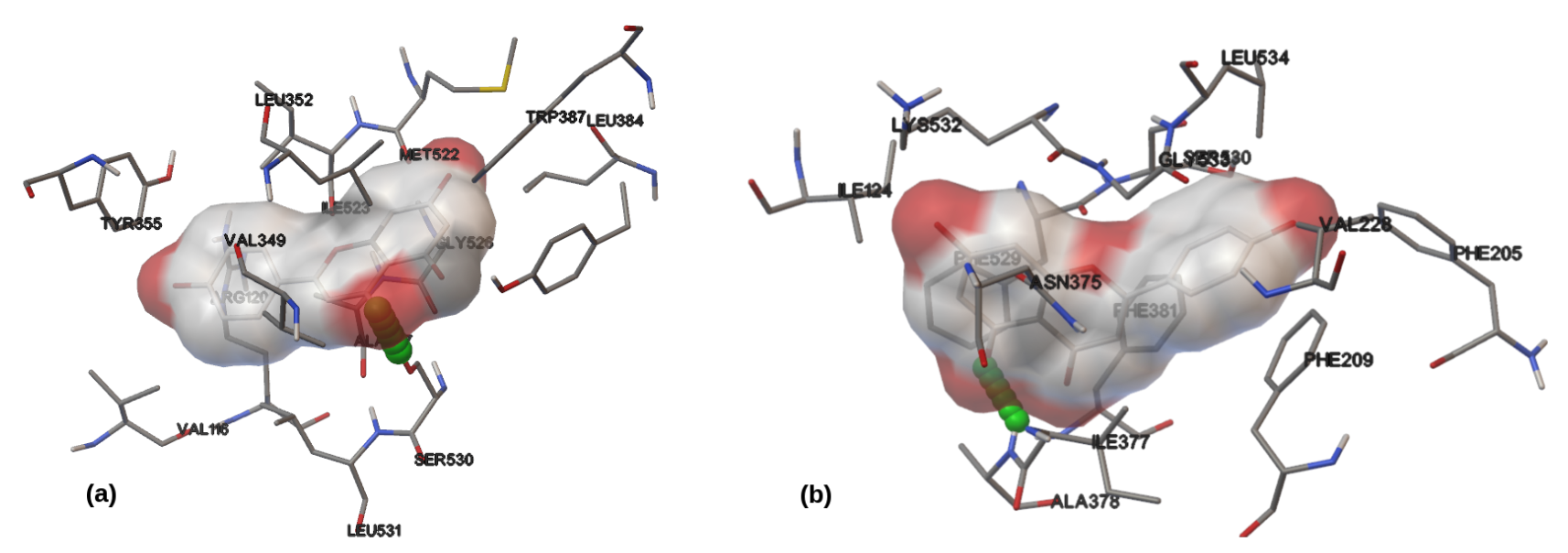

(c)
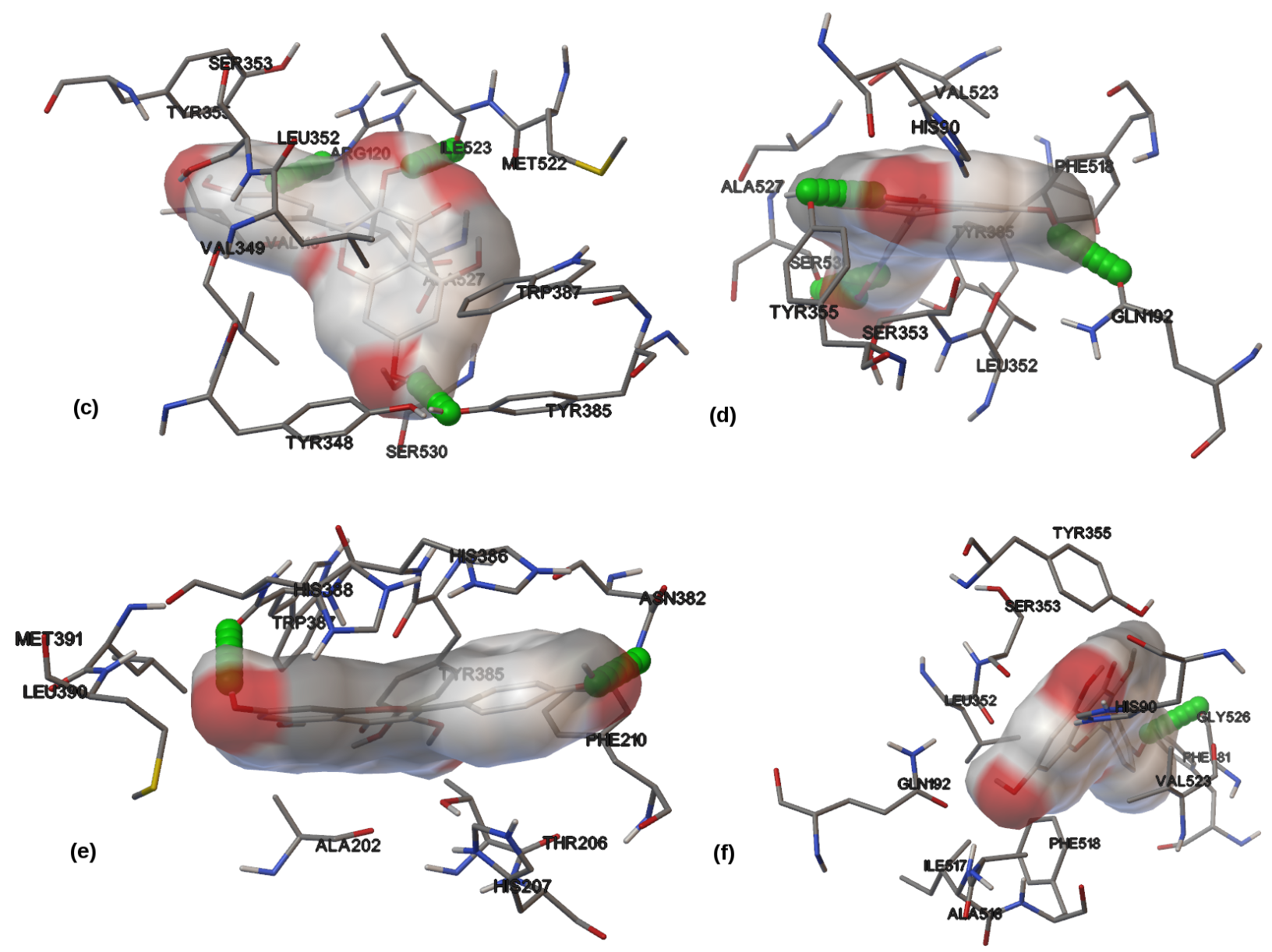

Figure 9: COX-Ligands complex. (a) COX-1/Apigenin system; (b) COX-2/Apigenin system; (c) COX-1/Quercetin system; (d) COX-2/Quercetin system; (e) COX-1/Kaempferol system; (f) COX$2 /$ Kaempferol system. In all complexes, the ligand is surrounded by amino acids of the enzyme active site. In green the hydrogen bonds formed.

\section{Selectivity Index of Protein Ligands SIPL}

The SIPL is a very important parameter to quantify the potential that some ligands have to inactivate a enzyme. This index shows the affinity for one or another isomeric form of COX in numerical and relative form. 
The ISPL is calculated by dividing the binding energy of each COX-2/ligand interaction between the binding energy of each COX-1/ligand interaction, taking into account the correspondence between the ligands.

The ISPL is defined as follows:

$$
I S=\frac{\frac{E_{i}, C O X 2-E_{\min }, C O X 2}{E_{\text {hig }}, C O X 2-E_{\min }, C O X 2}}{\frac{E_{i}, C O X 1-E_{\min }, C O X 1}{E_{\text {hig }}, C O X 1-E_{\min }, C O X 1}}
$$

Quercetin was the ligand with a higher SIPL in the active sites of both enzymes, followed by Galangin, Morin, Apigenin, Luteolin and Kaempferol.

Figure 10 shows the ISPL in a descent form. First, Quercetin have the higher value of selectivity with 1.17, while Kaempferol shows the lowest value with 0.91 . Finally, we can notice that the difference in the ISPL values is not very marked among the ligands, which is very notable in the values of the stability index where the difference is greater.

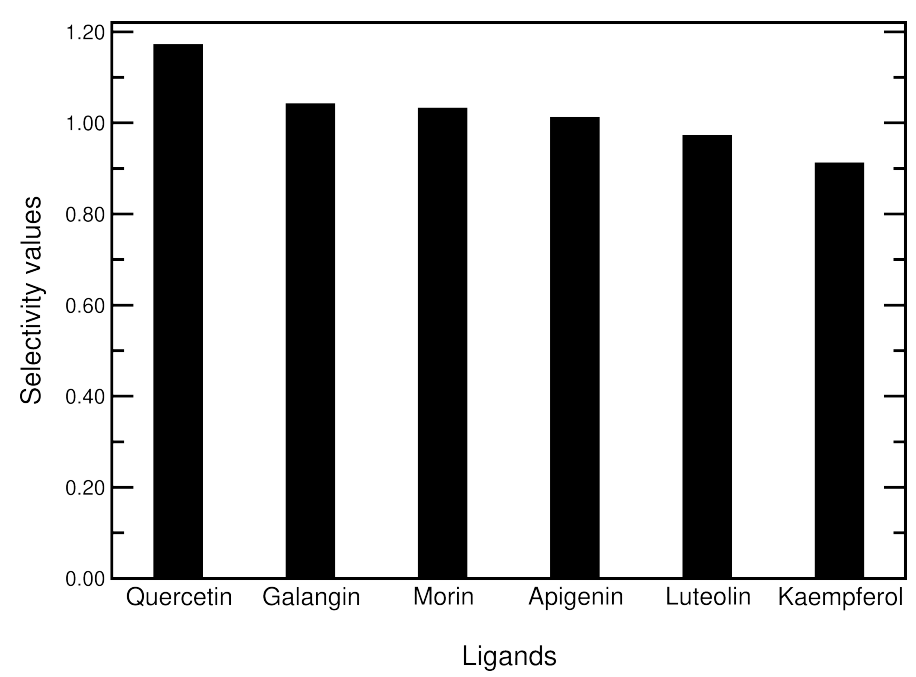

Figure 10: Selectivity index of ligands.

\section{Conclusions}

Las interacciones de los flavonoides con los aminoácidos del sitio activo de las enzimas se dan principalmente a través de los grupos hidroxilos que presentan en su estructura. El número de dichos grupos - $\mathrm{OH}$ varían de acuerdo al tipo de flavonoide, pero es importante notar que un metabolito por tener más grupos -OH que otro no lo hace más estable o selectivo.

- Los Puentes de Hidrógeno, aunque son un tipo de interacción muy importante, no definen la estabilidad de los ligandos durante el el acople, es decir que el hecho que un metabolito forme mayor 
número de enlaces de hidrógenos que otro no garantiza que siempre será el más estable.

- Con base a lo anterior, es de resaltar que tanto la estabilidad como la selectividad de los ligandos son parámetros independientes, es decir, que el hecho que un ligando sea estable en el sitio activo de la enzima no quiere decir que también sea muy selectivo por la misma; esto se puede ver al comparar las gráficas de estabilidad y selectividad donde para el primer caso, la apigenina presentó un mayor índice de estabilidad que el resto de los ligandos, lo cual no sucedió en el índice de selectividad.

\section{Supplementary Information SI}

\section{Acknowledgments}

\section{References}

1. Combariza, A. F.; Sullivan, E.; Auerbach, S. M.; Blanco, C. ; J. Phys. Chem. B. 2005, 109, 18439.

2. Combariza, A. F.; Gomez, D. A.; Sastre, G. ; Chem. Soc. Rev. 2013, 42, 114.

3. Marti, S.; Moliner, V.; Andres, J.; Roca, M.; Lopez-canut, V.; Silla, E.; Tunon, I.; Bertran, J.; An. Quim. 2011, 107, 144.

4. Combariza, A. F.; Sastre, G.; J. Phys. Chem. C. 2011, 115, 13751.

5. Lippow, S. M.; Tidor, B.; Curr. Opin. Biotechnol. 2007, 18, 305.

6. Paquet, E.; Viktor, H. L.; Biomed Res. Int. 2015, 2015, 1.

7. Ferreira, L. G.; Dos Santos, R. N.; Oliva, G.; Andricopulo, A. D. ; Molecules. 2015, 20, 13384.

8. Levine, I.; Rodriguez, A.; Pascual, A.; Roman, J.; Quantum Chemistry, 6th ed.; Pearson Education: New York, USA, 2009.

9. Perez, J. D.; J. Trop. Eng. 2014, 24, 117.

10. Kitchen, D. B.; Decornez, H.; Furr, J. R.; Bajorath, J.; Nat. Rev. Drug Discov. 2004, 3, 935.

11. Fitzpatrick, F. A.; Curr. Pharm. Des. 2004, 10, 577. 
12. Rouzer, C. A.; Marnett, L. J.; J. Lipid Res. 2009, 50 Suppl, S29.

13. Limongelli, V.; Bonomi, M.; Marinelli, L.; Gervasio, F. L.; Cavalli, A.; Novellino,, E.; Parrinello, M.; Proc. Natl. Acad. Sci. U. S. A. 2010, 107, 5411.

14. Garavito, R. M.; DeWitt, D. L.; Biochim. Biophys. Acta. 1999, 1441, 278.

15. Mohammed, M. S.; Osman, A. J.; Garelnabi, E. A.; Osman, Z.; Osman, B.; Khalid, H. S.; Mohamed, M. A.; Osman, J. A. J. Phytopharm. 2014, 3, 275.

16. Aswad, M.; Rayan, M.; Abu-Lafi, S.; Falah, M.; Raiyn, J.; Abdallah, Z.; Rayan, A.; Inflamm. Res. 2018, 67, 67.

17. Kumar, S.; Pandey, A. K.; Kumar, S.; Pandey, A. K. ; Sci. World J. 2013, 2013, 1.

18. Chen, H. J.; Chung, C. P.; Chiang, W.; Lin, Y. L.; Food Chem. 2011, 126, 1741.

19. https: //www.rcsb.org/, accesed in June 2018.

20. Wishart, D. S.; Feunang, Y. D.; Guo, A. C.; Lo, E. J.; Marcu, A.; Grant, J. R.; Sajed, T.; Johnson, D.; Li, C.; Sayeeda, Z.; Assempour, N.; Iynkkaran, I.; Liu, Y.; Maciejewski, A.; Gale, N.; Wilson, A.; Chin, L.; Cummings, R.; Le, D.; Pon, A.; Knox, C.; Wilson, M.; Nucleic Acids Res. 2018, 46, D1074.

21. Sidhu, R. S.; Lee, J. Y.; Yuan, C.; Smith, W. L.; Biochemistry. 2010, 49, 7069.

22. Picot, D.; Loll, P. J.; Garavito, R. M.; Nature. 1994, 367, 243.

23. Kurumbail, R. G.; Stevens, A. M.; Gierse, J. K.; McDonald, J. J.; Stegeman, R. A.; Pak, J. Y.; Gildehaus, D.; Iyashiro, J. M.; Penning, T. D.; Seibert, K.; Isakson, P. C.; Stallings, W. C. Nature. 1996, 384, 644 .

24. Drenth, J.; Mesters, J.; Principles of protein X-ray crystallography, 3th ed.; Springer New York: New York, USA, 2007.

25. Humphrey, W.; Dalke, A.; Schulten, K.; J. Mol. Graph. 1996, 14, 33.

26. Yuan, S.; Chan, S. H.; Hu, Z.; Wiley Interdiscip. Rev. Comput. Mol. Sci. 2017, 7, e1298.

27. http: //www. chemspider.com/, accessed in June 2017. 
28. Pence, H. E.; Williams, A.; J. Chem. Educ. 2010, 87, 1123.

29. www. chemspider. com/Chemical-Structure.4444100.html, accessed in June 2018.

30. www. chemspider. com/Chemical-Structure.4444102.html, accessed in June 2018.

31. Feng, B. Y.; Toyama, B. H.; Wille, H.; Colby, D. W.; Collins, S. R.; May, B. C.; Prusiner, S. B.; Weissman, J.; Shoichet, B. K.; Nat. Chem. Biol. 2008, 4, 197.

32. www. chemspider.com/Chemical-Structure.4444989.html, accessed in June 2018.

33. www. chemspider.com/Chemical-Structure.4444935.html, accessed in June 2018.

34. www. chemspider.com/Chemical-Structure.4444395.html, accessed in June 2018.

35. Cuevas, G.; Cortes, F.; Introduccion a la quimica computacional, 1ra ed.; Fondo de Cultura Economica: Mexico, 2003.

36. Foresman, J. B.; Frisch, Ae.; Exploring Chemistry With Electronic Structure Methods, 3th ed.; Gaussian, Inc.: Wallingford, CT, 2015.

37. Schlegel, H. B.; Wiley Interdiscip. Rev. Comput. Mol. Sci. 2011, 1, 790.

38. Zheng, J.; Frisch, M. J.; newblock J. Chem. Theory Comput. 2017, 13, 6424.

39. Schlegel, H. B.; J. Comput. Chem. 2003, 24, 1514.

40. http://openmopac.net/, accessed in July 2018.

41. Stewart, J. J.; J. Comput. Aided. Mol. Des. 1990, 4, 1.

42. Boyd, D. B.; Smith, D. W.; Stewart, J. J. P.; Wimmer, E.; J. Comput. Chem. 1988, 9, 387.

43. Stewart, J. J.; J. Mol. Model. 2004, 10, 155.

44. Dewar, M. J.; Thiel, W.; J. Am. Chem. Soc. 1977, 99, 4899.

45. http://vina. scripps.edu/, accessed in July, 2018).

46. Trott, O.; Olson, A. J.; J. Comput. Chem. 2009, 31, NA. 
47. Morris, G. M.; Goodsell, D. S.; Halliday, R. S.; Huey, R.; Hart, W. E.; Belew, R. K.; Olson, A. J.; J. Comput. Chem. 1998, 19, 1639.

48. Fuhrmann, J.; Rurainski, A.; Lenhof, H.-P.; Neumann, D.; J. Comput. Chem. 2010, 31, NA.

49. DeWitt, D. L.; El-Harith, E. A.; Kraemer, S. A.; Andrews, M. J.; Yao, E. F.; Armstrong, R. L.; Smith, W. L. ; J. Biol. Chem. 1990, 265, 5192.

50. Lecomte, M.; Laneuville, O.; Ji, C.; DeWitt, D. L.; Smith, W. L.; J. Biol. Chem. 1994, 269, 13207.

51. Mancini, J. A.; Riendeau, D.; Falgueyret, J. P.; Vickers, P. J.; O’Neill, G. P.; J. Biol. Chem. 1995, $270,29372$.

52. Rieke, C. J.; Mulichak, A. M.; Garavito, R. M.; Smith, W. L.; J. Biol. Chem. 1999, 274, 17109.

53. Zarghi, A.; Arfaei, S. Iran. J. Pharm. Res. 2011, 10, 655. 